\title{
Control of bulk and surface radioactivity in bolometric searches for double-beta decay
}

\author{
M. Pavan ${ }^{1,2, a}$, C. Callegaro ${ }^{1,2}$, S. Capelli ${ }^{1,2}$, M. Carrettoni ${ }^{1,2}$, M. Clemenza ${ }^{1,2}$, L. Gironi ${ }^{3}$, P. Gorla ${ }^{3}$, C. Maiano ${ }^{1,2}$, \\ C. Nones ${ }^{1,5}$, and M. Pedretti ${ }^{4,2}$ \\ 1 Università degli Studi di Milano-Bicocca, Milano, Italy \\ 2 INFN-Sezione di Milano-Bicocca, Milano, Italy \\ 3 INFN, Laboratori Nazionali del Gran Sasso, Italy \\ 4 Università dell'Insubria, Dipartimento di Fisica e Matematica, Como, Italy \\ ${ }^{5}$ Centre de Spectrométrie Nucléaire et de Spectrométrie de Masse, Orsay, France
}

Received: 13 December 2007 / Revised: 14 March 2008

Published online: 13 May 2008 - C Società Italiana di Fisica / Springer-Verlag 2008

Communicated by E. Bellotti

\begin{abstract}
The problem of background reduction in bolometers used to search for $\beta \beta(0 \nu)$ is reviewed with particular emphasis on bulk and surface radioactive contamination of the detectors.
\end{abstract}

PACS. 29.40.-n Radiation detectors - 23.40.-s $\beta$ decay; double $\beta$ decay; electron and muon capture

\section{Introduction}

Presently, $\mathrm{TeO}_{2}$ bolometers are among the best competitors to Ge diodes for the next-generation neutrinoless double-beta decay $(\beta \beta(0 \nu))$ experiments [1], a field in which - as for dark-matter detection - these devices are already giving remarkable results $[2-6] . \beta \beta(0 \nu)$ is one of the physical processes that can give access to the measurement of the absolute neutrino mass scale [7]. Indeed, if neutrino is a Majorana massive particle, then $\beta \beta(0 \nu)$ (in which an isotope $(A, Z)$ decays into its $(A, Z+2)$ isobar) is possible and becomes the main decay channel for a group of isotopes whose single-beta decay is forbidden. The decay is detected on the basis of the two electrons signal: given the negligible energy of the recoiling nucleus the sum kinetic energy of the two electrons is equal to the $Q$-value of the $\beta \beta(0 \nu)$ transition that, for the most interesting and studied isotopes, is at energies of few $\mathrm{MeV}$. The decay rate measures the half-life for $\beta \beta(0 \nu)$ of the studied isotope, and the square root of the half-life is inversely proportional to the so-called Majorana mass of the neutrino, which is a combination of the neutrino mass eigenvalues [7].

Bolometers are very sensitive calorimeters which measure the energy deposited by a single interacting particle through the corresponding temperature rise. These detectors belong to the class of solid-state detectors and share with Ge diodes the capability of reaching quite highenergy resolutions with sizable active mass devices. Both

\footnotetext{
a e-mail: pavan@mib.infn.it
}

these characteristics are of primary importance in $\beta \beta(0 \nu)$ experiments. When used for $\beta \beta(0 \nu)$ search, bolometers allow the calorimetric ( source $=$ detector $)$ approach: the isotopes candidate to the decay are part of the active mass of the detector. The latter is made of two elements: a single crystal (its molecule being a compound of the $\beta \beta(0 \nu)$ active nucleus) that plays the role of the calorimetric mass, and a sensor that measures the amount of energy released in the calorimetric mass, transducing the phonon signal into an electrical one. As an example, the presently running CUORICINO experiment uses $\mathrm{TeO}_{2}$ crystals $\left({ }^{130} \mathrm{Te}\right.$ is a $\beta \beta(0 \nu)$ candidate) coupled to thermistors whose electrical conductance is modified by the crystal temperature rise. 62 such detectors are mounted in a copper structure to form the $40.7 \mathrm{TeO}_{2} \mathrm{~kg}$ CUORICINO array [2].

Unlike other solid-state detectors, bolometers are not ionization but phonon (or thermal) detectors. As a consequence, they are almost equally sensitive to any kind of particle interaction despite the way the energy is released. This means electrons, alpha-particles and nuclear recoils, depositing in the detector the same amount of energy, produce a pulse with the same amplitude (and shape). This property and the absence of a surface dead layer (and their operation in large arrays kept in vacuum) make the study of background sources in bolometers a little bit different from other, more traditional, detectors.

CUORE is a $\beta \beta(0 \nu)$ large-mass experiment based on a large-mass bolometric array of $\mathrm{TeO}_{2}$ detectors that will be constructed and operated within few years. In order to reach the sensitivity required to investigate the neutrino inverted hierarchy mass region (corresponding to a 
Majorana mass of about $50 \mathrm{meV}$ ) CUORE has to reach an extremely low background level. As a consequence, an important program of R\&D was started by the CUORE Collaboration aiming at the identification and reduction of background sources. Some of the results obtained so far are here summarized.

\section{Background sources}

The search for a rare event, such as a $\beta \beta(0 \nu)$, means operating a detector removing, as far as possible, any spurious source that can mimic the rare event (e.g., radioactive sources or cosmic rays). The residual background counting rate measures the sensitivity of the experiment. In a calorimetric experiment the signature of a $\beta \beta(0 \nu)$ decay is a peak appearing, in the spectrum measured by the detector, at the transition energy ( $Q$-value). This peak corresponds to the deposition of the whole energy released by the two electrons produced in the decay (the probability of electron escape in typical $\beta \beta(0 \nu)$ calorimetric detectors is indeed quite small). Any event producing an energy deposition similar to that of the $\beta \beta(0 \nu)$ decay increases the background and hence spoils the sensitivity of the experiment. Background sources are cosmic rays, natural or artificial radioactive contaminations in the laboratory environment and in the experimental set-up and finally the radioimpurities contained in the detector itself [8]. Cosmic rays and environmental radioactivity can be vetoed and/or shielded effectively as it will be for the next-generation $\beta \beta(0 \nu)$ calorimetric experiments $[1,6,9]$. In particular, cosmic rays are strongly reduced by operating the detectors in deep underground laboratories. In the case of CUORICINO, as it will be for CUORE, the underground site is the Laboratori Nazionali del Gran Sasso (LNGS) at a depth of about 3200 m.w.e.

The ultimate limit to the sensitivity becomes therefore the surface/bulk radioactivity of the detector themselves. There are three main kinds of radioactive isotopes:

1) a natural unstable nuclide decaying into a stable one (as ${ }^{40} \mathrm{~K}$ ) or isotopes that give birth to a radioactive chain as ${ }^{232} \mathrm{Th},{ }^{238} \mathrm{U}$ and ${ }^{235} \mathrm{U}$;

2) artificially radioactive nuclei such as those produced in nuclear power plants or by atmospheric nuclear explosions (e.g., ${ }^{134} \mathrm{Cs}$ and ${ }^{137} \mathrm{Cs}$ ), or those produced for medical, research and industrial purposes $\left(e . g .,{ }^{60} \mathrm{Co}\right)$

3) cosmogenic radioisotopes $\left(e . g .,{ }^{14} \mathrm{C},{ }^{7} \mathrm{Be}\right.$ in air, ${ }^{60} \mathrm{Co}$ and many others in copper, ${ }^{68} \mathrm{Ge}$ in germanium);

The $Q$-value for the most interesting $\beta \beta(0 \nu)$ candidates is at $2048 \mathrm{keV}$ for ${ }^{76} \mathrm{Ge}, 2530 \mathrm{keV}$ for ${ }^{130} \mathrm{Te}$, and above $2.6 \mathrm{MeV}$ for ${ }^{82} \mathrm{Se},{ }^{100} \mathrm{Mo},{ }^{116} \mathrm{Cd},{ }^{96} \mathrm{Zr}$ and ${ }^{150} \mathrm{Nd}$. Not all the radioactive isotopes can therefore contribute to background. Because of gamma emissions the most dangerous are ${ }^{232} \mathrm{Th}$ and ${ }^{238} \mathrm{U}$ daughters together with some cosmogenically activated isotopes that are specific of the detector considered (e.g., for $\mathrm{TeO}_{2}$ experiments this is ${ }^{60} \mathrm{Co}$, while for $\mathrm{Ge}$ experiment also ${ }^{68} \mathrm{Ge}$ is worrisome). Above $2.6 \mathrm{MeV}$ alpha emission plays a relevant role in the background composition. Once again the natural chains
Table 1. Radiopurity levels in ${ }^{232} \mathrm{Th}$ and ${ }^{238} \mathrm{U}$ required for $\mathrm{TeO}_{2}$ and $\mathrm{Cu}$ in CUORE. In parenthesis the sensitivities achievable with standard techniques for screening radioactivity.

\begin{tabular}{cccc}
\hline Element & ${ }^{232} \mathrm{Th}$ & ${ }^{238} \mathrm{U}$ & Units \\
\hline $\mathrm{TeO}_{2}$ crystal bulk & $0.5(100)$ & $0.1(100)$ & $\mathrm{pg} / \mathrm{g}$ \\
$\mathrm{TeO}_{2}$ crystal surf. & $1(100)$ & $1(100)$ & $\mathrm{nBq} / \mathrm{cm}^{2}$ \\
$\mathrm{Cu}$ holder bulk & $4(2)$ & $2(2)$ & $\mathrm{pg} / \mathrm{g}$ \\
$\mathrm{Cu}$ holder surf. & $1(100)$ & $1(100)$ & $\mathrm{nBq} / \mathrm{cm}^{2}$ \\
\hline
\end{tabular}

of ${ }^{232} \mathrm{Th}$ and ${ }^{238} \mathrm{U}$ predominate in the alpha emission recorded by the detectors, although other long-lived alphadecaying nuclei exist in nature.

\section{$3{ }^{232} \mathrm{Th}$ and ${ }^{238} \mathrm{U}$ background control and reduction}

In principle a preliminary check of the materials used for detector construction allows a relevant reduction of most natural/artificial contaminants and a reduced exposure to cosmic rays (through underground storage of materials) allows to get rid of the cosmogenic ones [8]. Detector handling in clean rooms, where Rn content has been significantly reduced, and surface treatment of the detector parts with ultraclean reagents, can generally prevent from recontamination of the detector parts after production. Where does this procedure fail? And why the background problem is widely agreed as "The Problem" of next-generation $\beta \beta(0 \nu)$ experiments? The answer is in the failure of the traditionally used techniques for material selections. To ensure the required radiopurity of materials, of reagents and of the environment, these are measured with high-purity germanium (HpGe) gamma spectroscopy, inductively coupled plasma mass spectrometry (ICP-MS), neutron activation and so on. But reaching the level of the order of $\mu \mathrm{Bq} / \mathrm{kg}$ - as often required in new experiments - for any contaminant and for any material is not straightforward. To have an idea of the importance of the problem the radiopurity levels required (in order to achieve a background counting rate lower than $10^{-2} \mathrm{c} / \mathrm{keV} / \mathrm{kg} / \mathrm{yr}$ ) for the main two materials to be used in CUORE [1] detector construction, $\mathrm{TeO}_{2}$ and $\mathrm{Cu}$, are reported in table 1 . In parenthesis the sensitivities usually achieved by conventional techniques [10].

As clearly shown, in a large fraction of cases conventional techniques are not enough to reach the desired sensitivity. The solution to the problem comes from the use of the detector itself as a device to measure its own radioactivity. The approach consists in realizing prototypes of the detectors to be employed in next-generation experiments and to use them to measure their own background. As an example in fig. 1 is shown a small CUORE prototype (named RAD) used to evaluate background achievements obtained after material selection and with different cleaning techniques. The RAD detector is an array of eight $790 \mathrm{~g} \mathrm{TeO}_{2}$ crystals mounted inside a copper holder and 


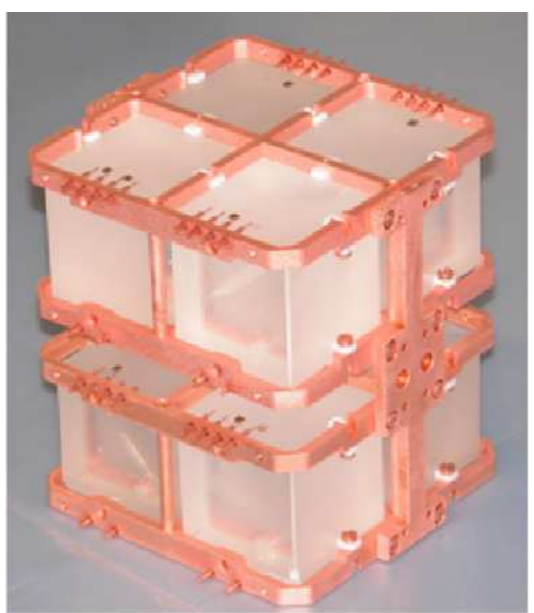

Fig. 1. (Colour on-line) A small bolometric array (named RAD) used for test purposes: an eight-crystal array mounted inside its $\mathrm{Cu}$ holder. The $\mathrm{TeO}_{2}$ crystals have a mass of $790 \mathrm{~g}$ each and the detector is completed by a $\mathrm{Cu}$ full coverage here removed.

operated in the refrigerator installed in Hall C of LNGS. The refrigerator is externally shielded with a lead and a paraffin $+\mathrm{H}_{3} \mathrm{BO}_{3}$ layer to reduce the environmental gamma and neutron fluxes to negligible levels. In this way the detector measures a background highly dominated by its own radioactivity and with a not negligible gamma contribution coming from the refrigerator itself.

\subsection{Monte Carlo simulations}

In order to extract numerical information from the prototype measurement performed with a bolometric array, a Monte Carlo simulation, capable of reproducing the main features of the detector geometry and response, is needed (the procedure is therefore identical to the one that is applied to evaluate the radioactive content of samples measured with Ge diodes). GEANT4 [11] is a widely used package for particle generation and identification that allows a detailed description of any detector geometry. A simulation for large-mass bolometers, based on GEANT4 package was realized [12] for this purpose. The simulation contains a full description of the experimental set-up and of the detectors (e.g., RAD and CUORICINO) used for background studies in the context of the ILIAS (Integrated Double-beta-decay European Activities) project, as well as the full geometry of the future detector CUORE. This means that the external shields surrounding the cryostat, the cryostat itself (simply represented by its thermal shields), the copper holder of the detector, the crystals and the small parts present inside the detector copper holder (the Ge sensors glued on the crystals, their gold bonding wires, the PTFE - PolyTetraFluoroEthene - stands) are introduced in the simulation. Bulk and surface contaminations of each of the previously listed elements can be simulated. Radioactive decays are simulated within this code, taking into account the level population and the time structure of the decay chains. All the particles produced by a decay (including nuclear recoils) are generated and propagated.

By comparing the experimental data with the Monte Carlo simulation for the contamination of one of the elements of the detector, upper bound or values of contaminant activities in that element can be determined. Upper bounds are obtained evaluating the maximum activity compatible with the experimental data, this is a quite conservative (but safe) approach since it does not imply a background model. Depending on mass, location and surface/volume ratio of the element under consideration the upper bound is extracted from a different energy region of the spectrum. The identification and determination of values (and not bounds) for contaminant activities requires - on the contrary - a background model in order to ascribe a fraction of the measured counts to the source under consideration. This is done considering all the information coming from experimental data and comparing different possible hypotheses for the sources location and typologies. Whenever the signature of the measured background can be ascribed to only one element, the activity of that source can be computed.

\subsection{Bolometric measurement of radioimpurities}

In a bolometric array, bulk and surface contaminations of the crystals are clearly distinguishable from each other and from the background due to external contaminations (i.e. radioactive emission of the holder or of the set-up) or to cosmic rays. Bulk contaminations in alpha-decaying isotopes produce sharp peaks due to the energy deposition by both the alpha-particle and the recoiling nucleus. Thanks to the characteristic feature of bolometers of having a quenching factor near to one for any kind of particle, these peaks appear at the $Q$-value of the reaction. Surface contaminations of the crystals produce asymmetric long-tailed peaks due to the escape of part of the alpha (seldom the recoil) energy. Since in an array at least part of the bolometer surface is facing - in vacuum - a second bolometer, and since bolometers do not have a dead layer, the particle escaping the first crystal - heavy straggledhas a not null probability of depositing its remaining energy in the nearby one. In this case a characteristic pattern of coincidence is recorded. Bulk and surface contamination in gamma-decaying isotopes, can be recognized as well if the decaying nucleus has a beta emission or if more than one gamma-ray is emitted.

In this way crystals contaminations due to ${ }^{232} \mathrm{Th}$ and ${ }^{238} \mathrm{U}$, as well as their daughters out of secular equilibrium, can be measured (comparing the experimental spectrum with a Monte Carlo simulation as discussed in sect. 3.1) at the level of about $0.1 \mathrm{pg} / \mathrm{g}$ for bulk and $10 \mathrm{nBq} / \mathrm{cm}^{2}$ for surface operating a prototype detector like the RAD (8 crystals of $790 \mathrm{~g}$ each, 1 month measurement and a heavy shielded set-up).

What about contaminations coming from inert parts of the detector (the $\mathrm{Cu}$ holder or the other small parts)? If they are in the bulk and the inert material has a relevant 


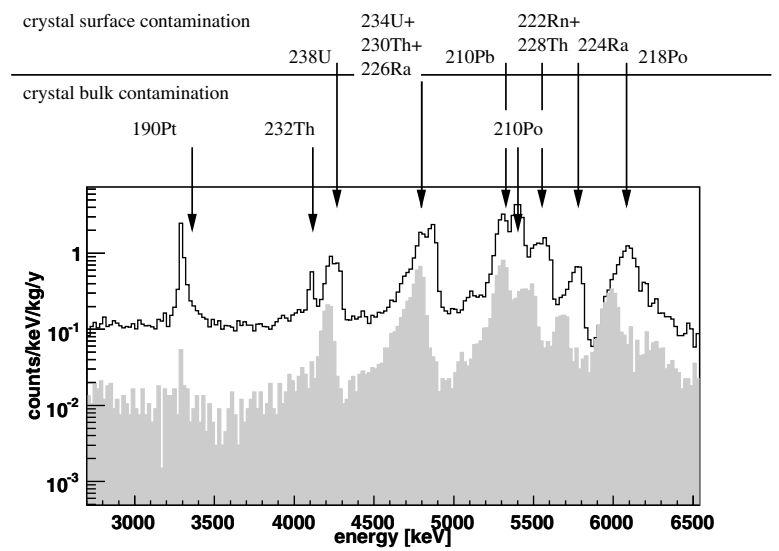

Fig. 2. Single-hit (black histogram) and double-hit (grey filled histogram) sum spectra of CUORICINO detectors. It is clearly evident that most peaks appearing in the single-hit spectrum have a correspondence in the double-hit spectrum: they are due to an alpha surface contamination in ${ }^{232} \mathrm{Th}$ and ${ }^{238} \mathrm{U}$ of the crystals. The exceptions is the peak centered at about $3.2 \mathrm{MeV}$ that is ascribed to a bulk contamination of the crystal in ${ }^{232} \mathrm{Th}$. The small amount of double-hit events in correspondence to the ${ }^{190} \mathrm{Pt}$ peak are due to spurious coincidences. The statistics here reported corresponds to the sum of the 42 CUORICINO $790 \mathrm{~g}$ crystals on a $\sim 8400$ hours background measurement.

mass (of the same order of magnitude of the crystals) the best evaluation of its contamination comes from the observation of the low-energy gamma peaks. These peaks must be due to a close contamination since otherwise they would be shielded. Comparing the Monte Carlo simulation of bulk contamination in the detector holder structure or in the thermal shields surrounding the detector with the experimentally measured background upper bounds on the contaminations are found. The sensitivity to a ${ }^{232} \mathrm{Th}$ and ${ }^{238} \mathrm{U}$ contamination can be as high as few $\mathrm{pg} / \mathrm{g}$, the same reached with the best $\mathrm{HPGe}$ (high efficiency+ultra low background cryostat) gamma spectrometry [10]. For small parts (in the case of the array shown in fig. 1 these are the PTFE stands, the pins, the glue ...) as well as for surface contaminations the best sensitivity on ${ }^{232} \mathrm{Th}$ and ${ }^{238} \mathrm{U}$ is obtained looking to their alpha background. In the case of extremely thin surface layers (below $0.01 \mu \mathrm{m}$ ) the straggling of the alpha-particle exiting the inert material and entering the crystals is so low that alpha peaks appear in the spectra recorded by the bolometers. This generally happens when the contamination is due to deposition (as for ${ }^{210} \mathrm{~Pb}$ content of dust) or to implantation (as when the surface contamination is due to the exposure of the material to a radioactive alpha source whose recoiling nuclei implant themselves into the material). With higher contamination thickness (or with bulk contaminations) the straggling of the alpha-particle is high and the crystal records a flat continuum.

As an example, fig. 2 shows the background measured by the CUORICINO array in the region above $2.6 \mathrm{MeV}$. Since here the environmental gamma contribution is negligible and the interaction due to neutron and muon yields a

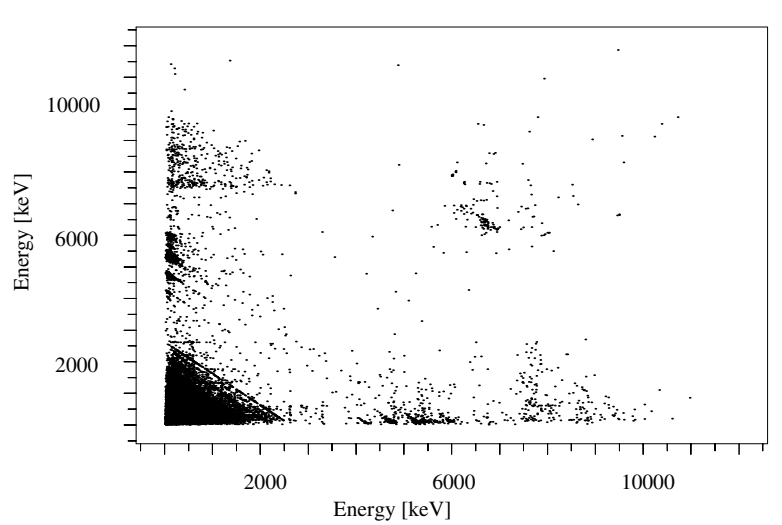

Fig. 3. CUORICINO background: scatter plot of double-hit events.

not prominent rate compared with the recorded rates, the background is dominated by alpha emissions. The spectra recorded by all the crystals are summed as if due to a single detector and different histograms are obtained applying a coincidence or an anticoincidence cut. To the $\mathrm{co}$ incidence (double-hit) spectrum belong events in which two crystals are contemporary hit: an alpha decay at the crystal surface produces an alpha-particle and a recoiling nucleus emitted back to back, one of the two can exit the crystal and enter the nearby one producing a double-hit event. It is worth to notice that, once summed together, the energies recorded by the two contemporary-hit crystals yield a monochromatic line at the $Q$-value. If the energies of double-hit events are plotted one against the other a characteristic scatter plot like the one in fig. 3 is obtained. In the anticoincidence (single-hit) spectrum appear events in which only one crystal was hit, either because all the particles emitted in the decay were fully contained, or because the one escaping entered an inert part of the detector (most likely the holder). Finally, also decays originating in the inert part of the detector, as it is for alpha contaminations in the $\mathrm{Cu}$ holder, contribute mainly to the single-hit spectrum. The high counting rate in CUORICINO anticoincidence spectrum in the region between 3 and $4 \mathrm{MeV}$ can be explained only by surface contamination of the inert part of the detector facing the crystals (mainly the copper holder). A Monte Carlo reconstruction of the CUORICINO measured background in the alpha region is reported in fig. 4 for both singleand double-hit histograms. This reconstruction is based on all the identified radioactive sources (i.e. all those that give clear structures in the single- or double-hit spectrum). These sources do not account for most of the flat background measured between 3 and $4 \mathrm{MeV}$, this background is therefore ascribed to a thick surface contamination in ${ }^{238} \mathrm{U}$ or ${ }^{232} \mathrm{Th}$ or ${ }^{210} \mathrm{~Pb}$, more likely located on the $\mathrm{Cu}$ holder (since this is the largest surface facing the crystals). In the reported Monte Carlo reconstruction the surface contaminations in ${ }^{238} \mathrm{U}$ and ${ }^{232} \mathrm{Th}$ of the crystals are assumed to have a depth of about $1 \mu \mathrm{m}$, while a ${ }^{210} \mathrm{~Pb}$ $10 \mu \mathrm{m}$ contamination in the $\mathrm{Cu}$ crystal holder is assumed to explain the $3-4 \mathrm{MeV}$ flat background. 


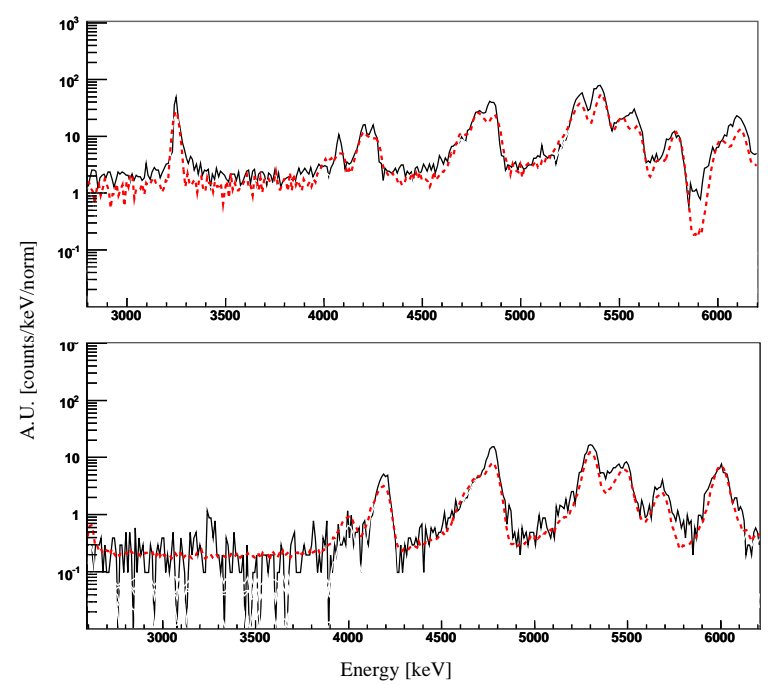

Fig. 4. (Colour on-line) Monte Carlo reconstruction of the CUORICINO measured background in the alpha region. The continuous line is the experimental data, the dotted line is the Monte Carlo simulation. In the upper panel is shown the single-hit spectrum and in the lower the double-hit spectrum. The hypotheses here are a ${ }^{238} \mathrm{U}$ and ${ }^{232} \mathrm{Th}$ surface contamination of the $\mathrm{TeO}_{2}$ crystals with a $1 \mu \mathrm{m}$ depth, a bulk crystal contamination in ${ }^{190} \mathrm{Pt}$ and ${ }^{210} \mathrm{Po}$ and a ${ }^{210} \mathrm{~Pb} 10 \mu \mathrm{m}$ depth contamination on the copper holder. This last source is the one with the weakest signature since other alpha contaminations (in ${ }^{238} \mathrm{U}$ or ${ }^{232} \mathrm{Th}$ ) of similar thicknesses yield equivalent contributions.

\subsection{Impurities reduction in $\mathrm{TeO}_{2}$ crystals}

The technique described in the previous section, in which a small prototype is used to evaluate the radioactive quality of detector materials, when joined with a pre-screening of the components with traditional techniques, allows to reach efficiency and extremely high sensitivities. In the case of $\mathrm{TeO}_{2}$ crystal arrays this procedure was applied to the optimization of crystal growth and of crystal surface treatment. The RAD detector (or similar detectors operated like RAD in hall $\mathrm{C}$ ) was used to check results. The main information and achievements obtained in this study are summarized here [13]:

- Crystal growth techniques: crystal growth with Bridgman method as by SICCAS (Shanghai Institute of Ceramics, Chinese Academy of Sciences, China) guarantees an impurity segregation that reduces by $3 / 4$ orders of magnitude the ${ }^{232} \mathrm{Th}$ and ${ }^{238} \mathrm{U}$ content, apparently this is not true if the Czochralsky method as by CTI (Crystal Technology Inc., CA, USA) is used. This was proved with a bolometric measurement mounting in a RAD-like array $4 \mathrm{TeO}_{2}$ crystals grown by SICCAS and 4 grown by CTI. Both the two types of crystals were produced with selected powders whose contamination in ${ }^{232} \mathrm{Th}$ and ${ }^{238} \mathrm{U}$ was measured. While in the case of SICCAS crystals a large reduction of the total activity was observed, in the case of CTI crystals the same reduction factor was not observed. In both cases crystal contaminations were evaluated on the basis of the intensities of ${ }^{238} \mathrm{U}$ and ${ }^{232} \mathrm{Th}$ alpha peaks.

- Crystal growth protocol: monitoring the entire crystal production chain, selecting materials and reagents by gamma spectroscopy and ICPMS, can guarantee the achievement of contamination levels as low as $0.01 \mathrm{pg} / \mathrm{g}$ in ${ }^{232} \mathrm{Th}$ and ${ }^{238} \mathrm{U}$. This was proved again by a bolometric measurement (with RAD) of SICCAS crystals grown according to such a protocol. Crystal contaminations were evaluated on the basis of the intensities of ${ }^{238} \mathrm{U}$ and ${ }^{232} \mathrm{Th}$ alpha peaks.

- Surface mechanical treatment: once grown, crystals are cut and mechanically treated on the surface in order to obtain the required dimensions and shape. This process, as performed by SICCAS, introduces a dangerous surface contamination of crystals that have to be removed. A re-treatment of the crystal surface with radiopure powders (with contaminations certified by gamma spectroscopy to levels below $100 \mathrm{pg} / \mathrm{g}$ ) is not enough to guarantee an improvement of the radioactive quality of the crystals surfaces (probably due to recontamination by the removed materials). Indeed CUORICINO $\mathrm{TeO}_{2}$ crystals were treated by SICCAS with contaminated (measured) powders and were later re-treated in Italy with ultrapure lapping materials, nonetheless they show a strong surface contamination as clearly evident from figs. 2 and 3.

- Chemical etching: the residual contamination due to crystal cut as well as crystal recontamination by handling and polishing or exposure to dust/air can be removed (to levels of the order of $\mathrm{nBq} / \mathrm{cm}^{2}$ ) by ultrapure acids etching (i.e. with ICPMS measured levels of the order of $\mathrm{pg} / \mathrm{g}$ ). This again was proved mounting in the RAD detector $\mathrm{TeO}_{2}$ crystals treated with ultrapure acids. The quite low surface contamination is however obtained at the price of a high roughness of the surface itself. The consequence being a deterioration of the detector performances.

- Chemical etching + mechanical treatment: an acid treatment of crystals followed by an accurate mechanical lapping can guarantee a surface contamination level of the order of $\mathrm{nBq} / \mathrm{cm}^{2}$ and a good surface quality. Once again a bolometric measurement was used to prove that. This is the case of the RAD detector whose crystals, coming form the same batch of the CUORICINO ones, were re-treated with utrapure acids + lapping and showed a reduction of surface alpha peaks by a factor 4 as visible in fig. 5 and fig. 6 .

\section{Cosmogenic activation control and reduction}

The problem of cosmogenic activation of materials is generally addressed with a completely different approach. When the activation is due to the environmental activity of the laboratory in which the experiment is located only passive and active vetoes can give solution to the problem. On the contrary, if the activation is the one 


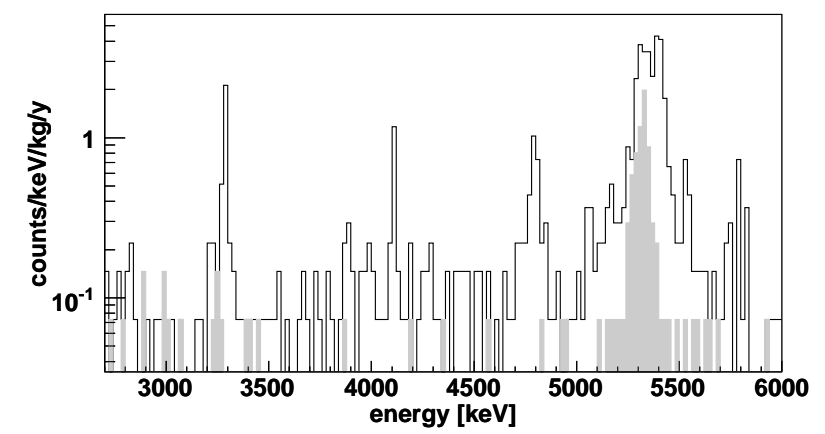

Fig. 5. Single-hit (black histogram) and double-hit (gray filled histogram) sum spectra of RAD detectors. If compared to fig. 2 the reduction of the crystal surface activity is clearly evident from the disappearance of alpha peaks. There are residual peaks in the single-hit spectrum that evidence an internal contamination in ${ }^{190} \mathrm{Pt}$ and ${ }^{232} \mathrm{Th}$. Only one peak is present in the double-hit spectrum, it is ascribed to a residual surface contamination in ${ }^{210} \mathrm{~Pb}$. The statistics here reported corresponds to 1 month with the RAD 8 -crystal array $(8 \times 790 \mathrm{~g})$.

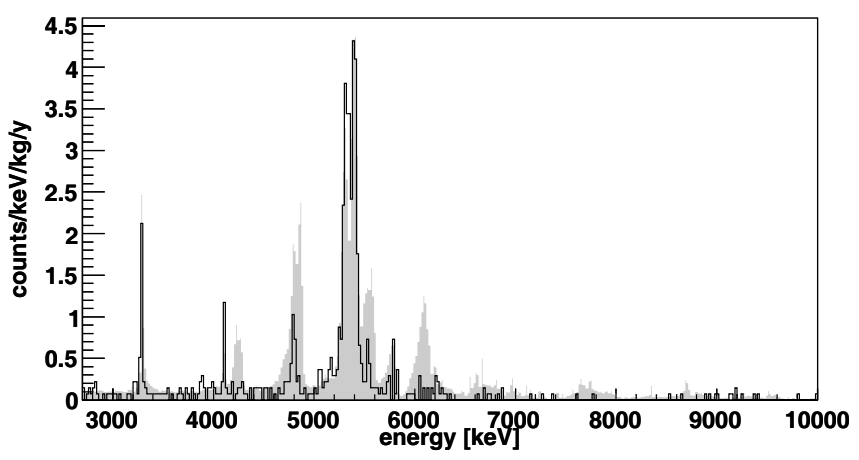

Fig. 6. Single-hit spectra of CUORICINO (gray filled histogram) and RAD (black histogram). The reduction of the crystal surface activity is clearly evident from the disappearance of the alpha peaks. There are residual peaks in the singlehit spectrum that evidence an internal contamination in ${ }^{190} \mathrm{Pt}$ and ${ }^{232} \mathrm{Th}$. Surface and bulk contaminations in ${ }^{210} \mathrm{~Pb}$ and ${ }^{210} \mathrm{Po}$ appear to be similar.

due to material exposure during production, machining and transportation to the site where the experiment takes place, exposure time reduction is the only solution. In both cases an inalienable ingredient is the knowledge of the activation cross-section for the materials of interest. When these cross-sections are not available (often only a theoretical evaluation of them can be found in the literature) the only solution is the realization of dedicated experiments in which the materials are irradiated at a neutron/muon beam. The present status of the experimental knowledge on activation cross-section is summarized in an IDEA status report [14-16]. On the basis of the result there included it was possible to establish - for example - the maximum exposure for $\mathrm{TeO}_{2}$ and $\mathrm{Cu}$ to be used for the CUORE detector.

\section{Active background rejection}

Procedure and techniques previously summarized deal with material selection and treatment for background re- duction. But a reduction of the detector spurious counting rate can be obtained also through active techniques. One, commonly used, consists in vetoing the particle interaction through a complementary detector, as in the case of muon vetoes used to reduce or cancel the muon-induced background. Other active techniques widely used are those based on particle discrimination obtained developing detectors capable of identifying the kind of particle that produced the event or the event topology $[5,3,6,9]$. In the case of bolometers one would like to be capable of removing the contributions from surface radioactivity, and from alphaparticles in general. This background source is particularly pernicious since, despite the ultra-clean procedures with which material surfaces are treated, recontamination is always a risk. There are two solutions that can be devised to the problem.

\subsection{Surface-sensitive bolometers}

The idea is to completely surround the bolometer with a thin alpha detector used as an anticoincidence shield. Any decay originating in the bolometer surface or in the alpha detector surface and depositing energy in both of them would be tagged and rejected. This would completely cancel the background coming from surface events. The practical realization of this kind of detector went through the construction of small surface-sensitive bolometers (SSBs) that could act as alpha detectors and could be operated at the same temperature of the main $\left(\mathrm{TeO}_{2}\right.$ in the case of the test done so far) big-size bolometer. Such detectors being very thin have an almost negligible gamma absorption, therefore, from the point of view of gamma interactions, they are completely transparent while they completely stop any alpha or recoiling nucleus. They shield the crystal from the surrounding and from their and its own surface activity. In actual realizations, to facilitate and simplify the mechanical structure of the actively shielded bolometer, the SSBs and the main crystal have been always maintained in thermal contact with each other [17]. In this case they do not act as an usual veto since any energy deposition in the SSB or in the main bolometer (called the Main in the following) produce a thermal signal on the whole system, but they act as pulse shapers. The shapes of the signal induced by a surface alpha, as seen by the SSB or by the main bolometer, are different from the signal due to an energy deposition that takes place only in the main bolometer or only in the SSB. In such a way the SSBs can also be used as passive shields acting simply as signal shapers. The preliminary results obtained with such composite detectors are impressive for their diagnostic potentialities: in fig. 7 the results obtained with a $\mathrm{TeO}_{2}$ test detector provided with 6 SSBs are shown. In the left panel the scatter plot of coincident events as measured by the Main and by the SSBs is shown. Signals produced by particles interaction in the Main are clearly identified as those belonging to the straight line (Main band in the following) in the lower part of the plot (they correspond to small-slow signals in the SSBs) while signals produced by particle interaction in the SSBs belong to the multi- 

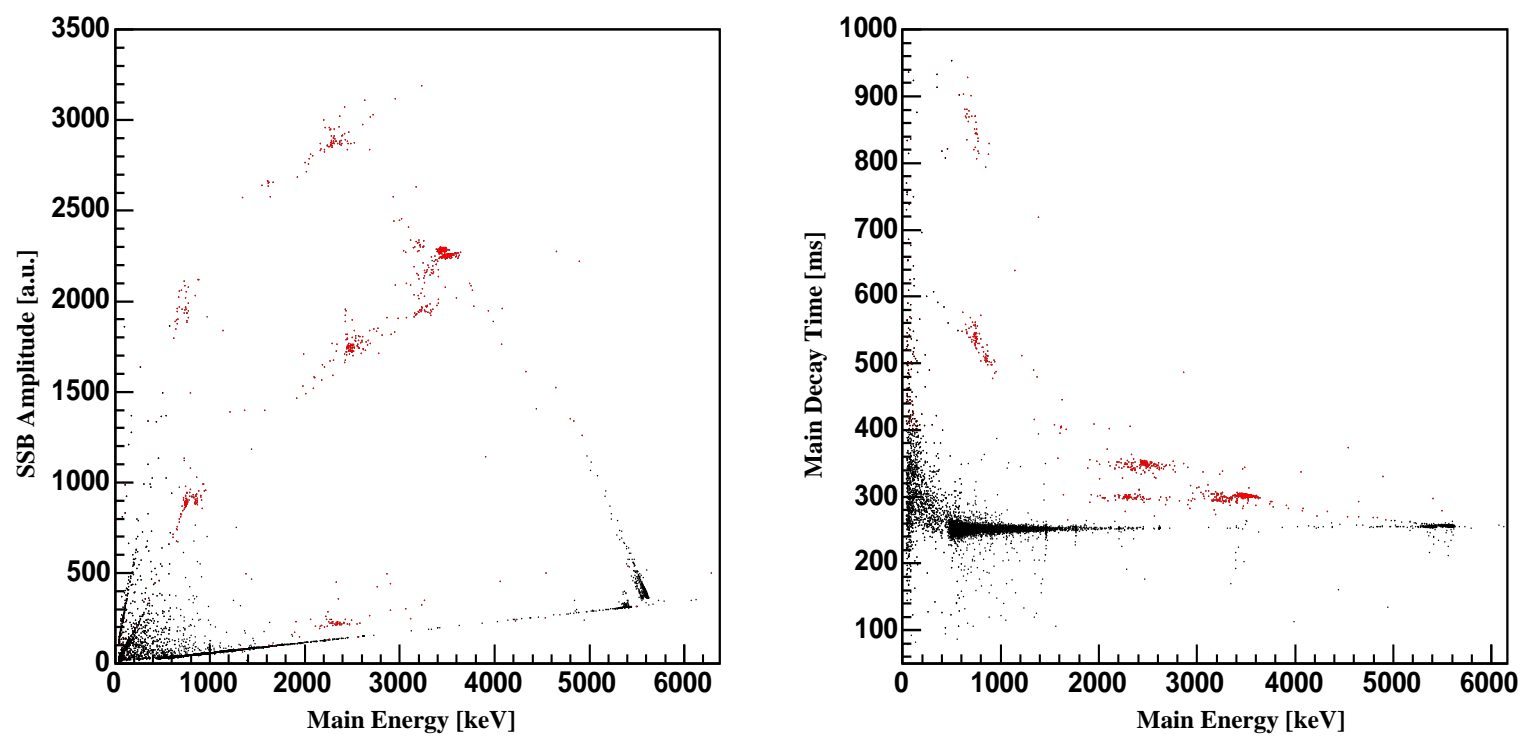

Fig. 7. (Colour on-line) Left panel: scatter plot of the pulse amplitudes recorded by SSB detectors (they are 6 but read-out in parallel) versus the one recorded by the Main. Right panel: scatter plot of pulse decay time versus pulse amplitude for the signals recorded by the Main. Signals rejected simply by appling a cut on the decay time of the pulses recorded by the Main are highlighted in both the scatter plots. It is clearly seen that in the $\beta \beta(0 \nu)$ region all the signals that in the left panel lay in the $S S B$ band or that are mixed events are rejected.

ple (because we have 6 different SSB detectors read out in parallel) curves ( $S S B$ band in the following) identified by a high SSB amplitude and low Main amplitude. When the energy of the particle is shared between the SSB and the Main, as it is for a surface alpha event, a line connecting the Main band and the $S S B$ band is produced (mixed events in the following). Few (probably two) similar lines are evident in the scatter plot and are ascribed to a surface alpha contamination between the slab and the crystal (more likely localized on the SSBs).

The right panel of fig. 7 shows, for the same events, the decay time versus amplitude as measured by the Main. What is clearly evident is that the events belonging to the Main band have the usual distribution while events that belong to the $S S B$ band or that are mixed events are clearly distinguishable having a much slower decay time. The conclusion is that the presence of the slabs thermally connected on the $\mathrm{TeO}_{2}$ crystal modify the response of the bolometer in such a way that bulk events are distinguishable from surface events just looking to their decay time. Indeed in fig. 7 the pulse rejection efficiency obtained by separating the bulk from surface events just using a cut on the Main decay time is shown by different colours. The rejection efficiency for alpha events, in the region of the $\beta \beta(0 \nu)$ signal, appears to be in both cases quite high.

\subsection{Scintillating bolometers}

$\mathrm{TeO}_{2}$ cannot be operated as a scintillation detector given the extremely low light yield of pure and doped samples [18], but scintillating crystals containing the more interesting $\beta \beta$ emitters have already been successfully tested as bolometers. The possibility of contemporary read-out of scintillation light (a signal highly sensitive to the ionizing properties of the interacting particle) and thermal signal (a signal practically insensitive to the type of particle) have been proposed and realized for the first time in the '90s expressively with the purpose of rejecting the alpha background in $\mathrm{CaF}_{2}$ crystals [19]. This technique is today used in dark-matter detection experiments [5] while its possible application for $\beta \beta(0 \nu)$ detection has been recently explored [20] testing different kind of crystals in Hall C. The successful results obtained for different composites (containing a $\beta \beta$ active isotope) and the high rejection capability for alpha background have opened new possibilities for the $\beta \beta(0 \nu)$ bolometric way.

\section{Conclusion}

A long work on background reduction in $\beta \beta(0 \nu)$ bolometric arrays is giving the first successful results. Both the passive reduction, by means of material selection and cleaning treatments, and the active reduction, by means of SSB or scintillating bolometers look highly promising for the future. Finally, the work done on the background results of large arrays like CUORICINO or small prototypes like RAD has improved a lot our knowledge of background sources yielding information that can be useful for the entire $\beta \beta(0 \nu)$ and dark-matter community.

This work has been supported by the ILIAS Integrated Double-beta-decay European Activities (Contract R113-CT2004-506222) as part of the EU FP6 program in Astroparticle Physics. 


\section{References}

1. C. Arnaboldi et al., Nucl. Instrum. Methods A 518, 775 (2004).

2. C. Arnaboldi et al., Phys. Rev. Lett. 95, 14501 (2005).

3. D.S. Akerib et al., Phys. Rev. D 73, 011102 (2006).

4. S. Fiorucci et al., Astropart. Phys, 28, 143 (2007).

5. G. Angloher et al., Astropart. Phys. 23, 325 (2005).

6. C.E. Aalseth et al., Nucl. Phys. B (Proc. Suppl.) 138, 217 (2005).

7. F.T. Avignone III, S.R. Elliott, J. Engel, Rev. Mod. Phys. 80, $481(2008)$

8. G. Heusser, Annu. Rev. Nucl. Part. C 45, 543 (1995).

9. I. Abt et al., Nucl. Instrum. Methods A 570, 479 (2004); S. Schonert et al., Nucl. Phys. (Proc. Suppl.) 145, 242 (2005); I. Abt et al., Eur. Phys. J. C 52, 19 (2007).

10. 2nd Topical Workshop in Low Radioactivity Techniques, http://lrt2006.in2p3.fr/Agenda.html.

11. GEANT4, http://geant4.web.cern.ch/geant4.
12. C. Bucci et al., Background study and Monte-Carlo simulations for large mass bolometers, in preparation.

13. CUORE Collaboration, LNGS Annual Report 2005 and LNGS Annual Report 2006, www.lngs.infn.it.

14. IDEA reports: Report_upgrade_codes, http://idea. dipscfm.uninsubria.it/frontend/docs/reports.

15. R. Silberberg, C.H. Tsao, Astrophys. J. 501, 911 (1998).

16. J. Martoff, P.D. Lewin, Comput. Phys. Commun. 72, 96 (1992).

17. C. Nones et al., Nucl. Instrum. Methods A 559, 355 (2006).

18. N. Coron et al., Nucl. Instrum. Methods A 520, 159 (2004).

19. A. Alessandrello et al., Nucl. Phys. B (Proc. Suppl.) 28A, 233 (1992); A. Alessandrello et al., Phys. Lett. B 420, 109 (1998).

20. S. Pirro et al., Proceedings of the Fifth International Conference on Non-Accelerator and New Physics (NANP2005), June 2005, Dubna, Russia (Pleiades Publishing Inc., 2005) arXiv::nucl-ex/0510074. 